\title{
Illusory Concomitant Motion in Ambiguous Stereograms: Evidence for Nonstimulus Contributions to Perceptual Organization
}

\author{
Mary A. Peterson \\ State University of New York at Stony Brook
}

\begin{abstract}
Three experiments were performed to test whether perceptual organization is cognitively or motivationally penetrable. In Experiments 1 and 2, subjects viewed a reversible stereogram while instructed to hold one depth organization. Responses about depth were recorded indirectly by recording responses about direction of the illusory concomitant motion that is perceptually coupled to depth in a stereogram. Inasmuch as perceptually coupled variables covary without necessary stimulus covariation, a postperceptual locus for any intention effects they exhibit is unlikely. Experiments 2 and 3 examined the possibility that instructed intention might influence perception indirectly by influencing eye movements: Viewers' vergence position was measured directly through responses about alignment of a vernier nonius fixation. In all three experiments, a residual effect of instructed intention was found. Therefore, instructed intention may influence perceptual organization by influencing internal nonstimulus components integral to the perceptual process.
\end{abstract}

A recurrent question about selective attention is whether it affects perceptual organization per se or whether it affects other systems such as memory or response (Haber, 1966; Hochberg, 1970; Kahneman \& Treisman, 1984; Lawrence \& LaBerge, 1956). Another issue is whether the selective aspect of attention is independent of eye movements. Recent paradigms avoid memory or response confounds by using detection (Posner, Snyder, \& Davidson, 1980) or search tasks (Schneider \& Shiffrin, 1977) and by obtaining speeded responses (Posner et al., 1980; Kahneman \& Treisman, 1984). These paradigms also show that shifts of attention need not be accompanied by saccadic eye movements (Posner et al., 1980); indeed, an attention shift to the left can accompany a planned saccade to the right (Remington, 1980). However, results obtained with detection or search tasks are difficult to apply to questions concerning the role of selective attention in the perceptual organization of objects (Kahneman \& Treisman, 1984; Peterson, 1984).

In order to examine selective effects in perceptual organization, Hochberg and Peterson (1979; Peterson \& Hochberg, 1983) defined selective attention more widely as the viewer's intention to fit a particular organization to a reversible stimulus. In their opposed-set procedure, intention was operationalized as straight-

This research was supported in part by a National Science Foundation Grant (BNS 82-09470) to Julian Hochberg and in part by a Public Health Service Award (2S07RR0706719) to the author. The first experiment was included in a dissertation submitted in partial fulfillment of the requirements for the PhD degree at Columbia University.

I thank Julian Hochberg for advice and guidance, and James Cutting, Barbara Dosher, William Epstein, Norma Graham, and two anonymous reviewers for constructive criticism. One of these reviewers suggested an analysis of the data that ultimately led to my devising the intention index. I appreciate the technical assistance provided by A. Castaldi, R. Reeder, and $\mathrm{H}$. Tepper.

Correspondence concerning this article should be addressed to Mary Peterson, Department of Psychology, State University of New York at Stony Brook, Stony Brook, New York 11794. forward instructions to viewers to try to hold one organization of a reversible figure. This operational definition of attention is compatible with early conceptions of selectivity in which perceived organization was a consequence of viewers' elective intentions to fit one or another mental structure to stimulus input (Helmholtz, 1866/1962; Mill, 1865). It also suits recent prescriptions for separating cognitive, or inferential, processes from noninferential, fixed, system components (Fodor \& Pylyshyn, 1981; Hochberg, 1981; Pylyshyn, 1981, 1984). As a methodological criterion for distinguishing the two, Pylyshyn suggests separating processes that are affected by viewers' knowledge or beliefs from those that are not, labeling these processes cognitively penetrable and cognitively impenetrable, respectively. The opposed-set procedure provides an index of motivational, rather than cognitive, penetrability inasmuch as effects on perceptual organization occur as a function of instructions to try to see one possible organization, rather than as a function of knowledge about the stimulus.

With the direct manipulation of intention through instruction, Peterson and Hochberg (1983; Hochberg \& Peterson, 1985) found quantitative evidence that instructed intention can affect the depth seen in a wire cube. The results do not appear to be mediated by overt eye movements, because intention effects obtained from viewers instructed to fixate an ambiguous region of a cube do not differ when a nearby region is biased toward or against instructed intention (Peterson \& Hochberg, 1983). Nor are the results easily attributed to postperceptual processes such as response bias: Indirect measures, obtained through reports about direction of oscillation of a randomly oscillating cube, agree with direct measures. The indirect measure was one that is perceptually coupled to depth so that when depth reverses, the perceived direction of rotation reverses as well. Perceptually coupled variables are unique indirect measures because they covary without stimulus covariation, provided an organization has been fitted to the display (Rock, 1983). In addition, they vary with what viewers really perceive, rather than with what they report perceiving, if the two differ (Gogel, 1976; Hochberg, 1956, 1974): For example, 
they remain unaffected when response biases concerning their paired variables are induced through hypnotic suggestion (Gogel, 1981; MacCracken, Gogel, \& Blum, 1980). Therefore, couplings seem to occur in perceptual processes, rather than in the stimulus or in postperceptual processes.

Therefore, these results converge on a model of perception in which the perceived organization is determined jointly by the viewer's intentions and by constraints in stimulus informationa motivationally penetrable system. However, they don't allow complete confidence about the level at which the effects of instructed intention occur. Even though overt eye movements may not be involved, other kinds of eye movements may still mediate both the attention effects and the perceptual coupling. For example, the convergence state of the eyes may vary across instruction, thereby providing different depth cues (Foley, 1980; Hay \& Sawyer, 1969) and modifying both the eye movements necessary to maintain fixation with self-movement (Post \& Leibowitz, 1982) and the motion interpretation fitted to the cube. If so, the opposed-set procedure may not be measuring the direct influence of intention on the perception of unchanging stimulation, but rather may be measuring the extent to which small eye movements are under conscious control.

Nor is it possible to be certain that the effects are not occurring in postperceptual processes. As Fodor (1983) has suggested for cognitive processes, motivation may affect only what is done with the output of an impenetrable perceptual system. In this case, early, impenetrable perceptual processes may register the relative shifting of the front and rear faces of an oscillating cube, but the labeling of the direction of motion may occur in a subsequent stage of processing. Instructed intention may affect this later stage rather than earlier stages of processing.

Thus, the two recurrent questions remain: How early are intention effects occurring? What are the potential contributions of eye movements? In the experiments reported here, these issues were explored further with a reversible stereogram as a stimulus. In a reversible stereogram, as in a wire cube, differential motion is coupled to changes in perceived depth, but here there is no relative retinal motion that might merely be registered early but labeled differentially later: Moving observers who see parts of a projected stereogram in depth perceive them to move relative to the surrounding regions, even though no relative displacement of near and far occurs between parts of the projected image. The perceived (but illusory) motion in a projected stereogram is in a direction opposite to the parallactic displacement that would have been provided by stationary real objects in depth. Furthermore, as can be seen in Figure 1, just as the direction of expected parallax for stationary objects nearer or farther than the plane of fixation varies, so the direction of the illusory motion differs, depending upon whether the region seen in depth relative to the rest of the display appears to be in front or in back. If intention instructions concerning depth affect responses about direction of illusory motion in a projected stereogram, where there is no relative motion subject to postperceptual interpretation, the possibility that intention effects occur postperceptually would be minimized.

Furthermore, when stereograms are used as stimuli, both large eye movements and smaller eye movements, such as vergence changes, can be controlled with a nonius fixation. In a nonius fixation, vertical line components are presented separately to each eye (Ogle, 1950). When vertically aligned on the plane of projection, these lines appear aligned only to a viewer whose eyes are converged on that plane. Thus, instructions to fixate a nonius and to maintain the alignment of its parts control both pursuit and vergence eye movements. Accordingly, in the experiments reported here, a nonius fixation is used both to keep fixation constant on the plane of projection and to measure any vergence changes that might be occurring.

In Experiment 1, reversible stereograms are used with the opposed-set procedure, and a nonius fixation is used to test whether intention instructions affect perceptual or postperceptual processes. In Experiments 2 and 3, direct measures of the perceived alignment of the nonius fixation are obtained to test whether the intention effects are mediated by vergence eye movements.

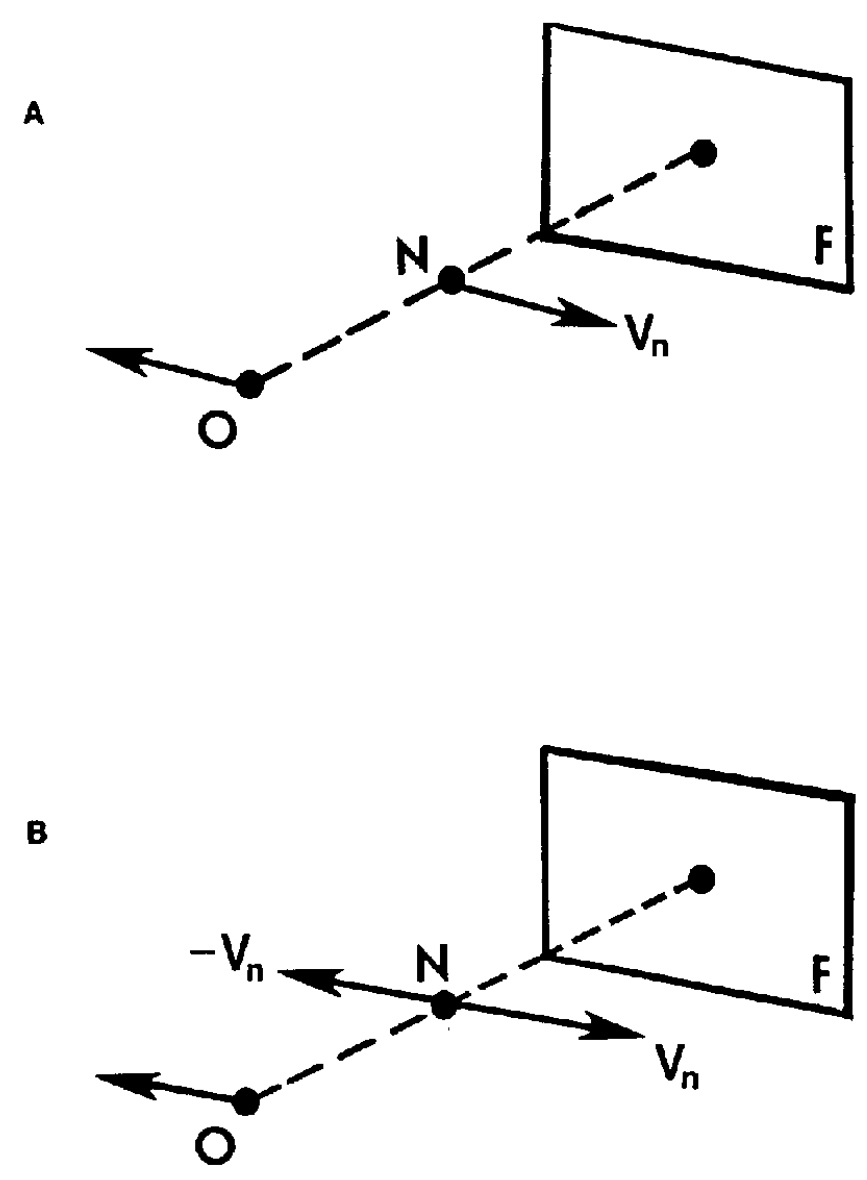

Figure 1. Panel A: Motion parallax information from a stationary, near object. (As an observer, [O], moves relative to a stationary object, $[\mathrm{N}]$, nearer than a plane, $[F]$, the retinal image of $\mathrm{N}$ displaces to the right relative to $F$. The shift for near objects is in the direction opposite to the observer's motion and is labeled $V_{n}$ in the figure. Despite this retinal placement, $\mathrm{N}$ is seen to be stationary.) Panel B: Motion parallax from a near object moving with velocity $-V_{n}$. (As the observer moves to the left, motion parallax $V_{n}^{\prime}$ is cancelled, leaving no net relative displacement of $\mathrm{N}$ and $\mathrm{F}$. Nevertheless, the observer sees the real motion. In the projected stereogram, no motion parallax, $V_{n}^{\prime}$ or real motion, $-V_{n}^{\prime}$ occurs. Yet the viewer perceives illusory motion in the direction of $-V_{n}$.) 


\section{Experiment 1}

In this experiment, the opposed-set procedure was used with reversible stereograms, containing central square regions that could appear either in front of or behind the surrounding region. Stimulus bias was varied across two stereograms: One was biased toward one depth organization; the other was unbiased. Subjects trying to hold the central region in one depth organization responded directly about depth on some trials and, on other trials, responded indirectly about depth through responses about illusory motion.

If instructed intention affects perceptual processes and not merely postperceptual processes, previous opposed-set effects should be replicated: Intention effects should be greater on the unbiased stereogram, and both depth responses and motion responses should vary as a joint function of hold instruction and stimulus bias.

\section{Method}

Subjects. Eight students at Columbia University volunteered to participate in this experiment. Six were graduate students and 2 were undergraduate students. All had normal or corrected-to-normal vision.

Materials. Stimuli were anaglyphic slides of reversible random dot stereograms (Figures 6.5-5* and 6.5-6* from Julesz, 1971), constructed by constraining the elements in a central square area of a random dot field so that the elements in the left eye's view were identical to elements shifted both to the left and to the right in the right eye's view (Julesz \& Johnson, 1968; Kaufman \& Pitblado, 1965). These shifted elements are in crossed and uncrossed disparity, specifying a central region in front of and behind the surround, respectively.

One anaglyph presented to the subjects was unbiased; the other was biased to favor one depth organization by changing $10 \%$ of the elements in the central square so as to fuse only when shifted in one direction. These two stereograms provided the stimulus bias manipulation in the opposed-set procedure (Peterson \& Hochberg, 1983).

The nonius fixation was a cross with its top and right arms seen by the right eye, and its bottom and left arms seen by the left eye. Each eye's view of the parts of the cross was positioned on a white disk located on the random dot surround just above the top edge of the reversible central region. These disks were superimposed in projection and served as a stimulus for convergence to the distance of the surround (the projection plane).

Stimuli were projected onto a screen at a distance of $156 \mathrm{~cm}$ from the subjects. The anaglyphs subtended $13.7^{\circ}$; the central square subtended $6.6^{\circ}$. The disparity of the central square was $18^{\prime}$ of arc for both crossed and uncrossed disparity.

Procedure. Subjects participated in the experiment individually. They began by viewing two unambiguous anaglyphs (one of crossed, the other of uncrossed, disparity) to become acquainted with depth in stereograms. Next, they were pretested so that appropriately "biased" and "unbiased" stimuli could be presented to each in the opposed-set procedure. This was necessary because, for viewers who prefer crossed or uncrossed disparity (Julesz, 1971; Kaufman \& Pitblado, 1965; Richards, 1970, 1971), physically unbiased stimuli are effectively biased toward their preference.

To determine preference, the biased anaglyph was presented twice, the second time with the right eye's view reversed for the left, which should switch the direction of bias. As a rule, subjects without depth preferences report seeing the central square at different depths before and after the images are reversed; subjects with depth preferences strong enough to override the physical biasing see the central square in the same depth despite the reversal of projection to each eye. In this study, 8 subjects showed a depth preference; two showed none.

Subjects with a depth preference were given three more presentations of bath the biased and the unbiased stereogram. On these presentations, 6 reported seeing the opposite organization for one of the physically biased (but not the unbiased) stereograms at least once. For these 6, then, the physically biased stimulus became the unbiased stimulus because it offered the possibility of reversal, and the physically unbiased stimulus became the biased stimulus. Bias was toward front for these subjects, and toward back for the other 2 subjects. (Two subjects who reported the same depth organization across 16 trials with the biased and the unbiased stereograms were dropped from the experiment at this point.)

Subjects then practiced reversing both stereograms for three 10-min sessions; two practice sessions occurred in 1 day and were separated by 3 to $4 \mathrm{hr}$. The next practice session occurred the next day. Immediately after this last practice session, subjects were given the opposed-set instructions: They were told that they would be participating in a series of trials on which they would view a succession of anaglyphs while trying to hold the central region in one depth organization. On some trials, they would be asked to hold the central square in front of the surround, and on other trials they would be asked to hold it behind. They were asked to do this solely by concentration: They were not to move their eyes in order to comply with the hold instructions, but were to fixate the nonius throughout. They were told that fixation was their primary instruction and that they should try to keep the parts of the fixation cross aligned and equally in focus throughout the trial.

Subjects participated in sixteen 30-s trials: four trials with each anaglyph with each hold instruction. Order of trials was counterbalanced. Before each trial, subjects were given the hold instructions for that trial and were reminded to fixate the nonius.

Subjects responded continuously during each trial by pressing one of two microswitches or by pressing neither. During the first eight trials, subjects viewed the anaglyph from a stationary position and responded directly about depth organization. On these trials, key presses indicated that the central region appeared in front of or behind the surround, and the absence of key presses indicated that the display looked flat. Subjects chose which microswitch represented front and which represented back. During the next eight trials, subjects rocked from side to side to a metronome beating at $0.77 \mathrm{~Hz}$ and responded about the direction of illusory motion. On these trials, subjects pressed keys only when the central square appeared to be moving. One key indicated that the central square appeared to be moving in the same direction as they were; the other key indicated that it moved in the direction opposite to their movement. Again, the subjects chose the mapping of response onto keys, and the absence of key pressing indicated no motion.

\section{Data Analysis}

Three measures were derived from the data. First, instructed responses, the mean duration that subjects reported seeing the instructed organization, were calculated directly from depth responses and indirectly from motion responses. These durations measure the viewers' success at seeing the instructed organization over other possible organizations.

Second, an intention index, $(I-U) /(I+U)$, was constructed from the relative distribution of time that subjects reported the instructed $(I)$ and uninstructed $(U)$ depth organizations on a given trial. This proportion ranges in value from -1 to +1 ; values greater than 0 indicate an effect of instructed intention. When this index is collapsed across intention instruction, it provides a measure of intention effects independent of observer bias toward front or back.

Third, flat time, the duration that the stereogram looked flat, was derived from the duration that neither key was pressed on both depth and motion trials. That is, both responses of no-depth and of no-motion were taken to mean that the stereogram looked flat. 


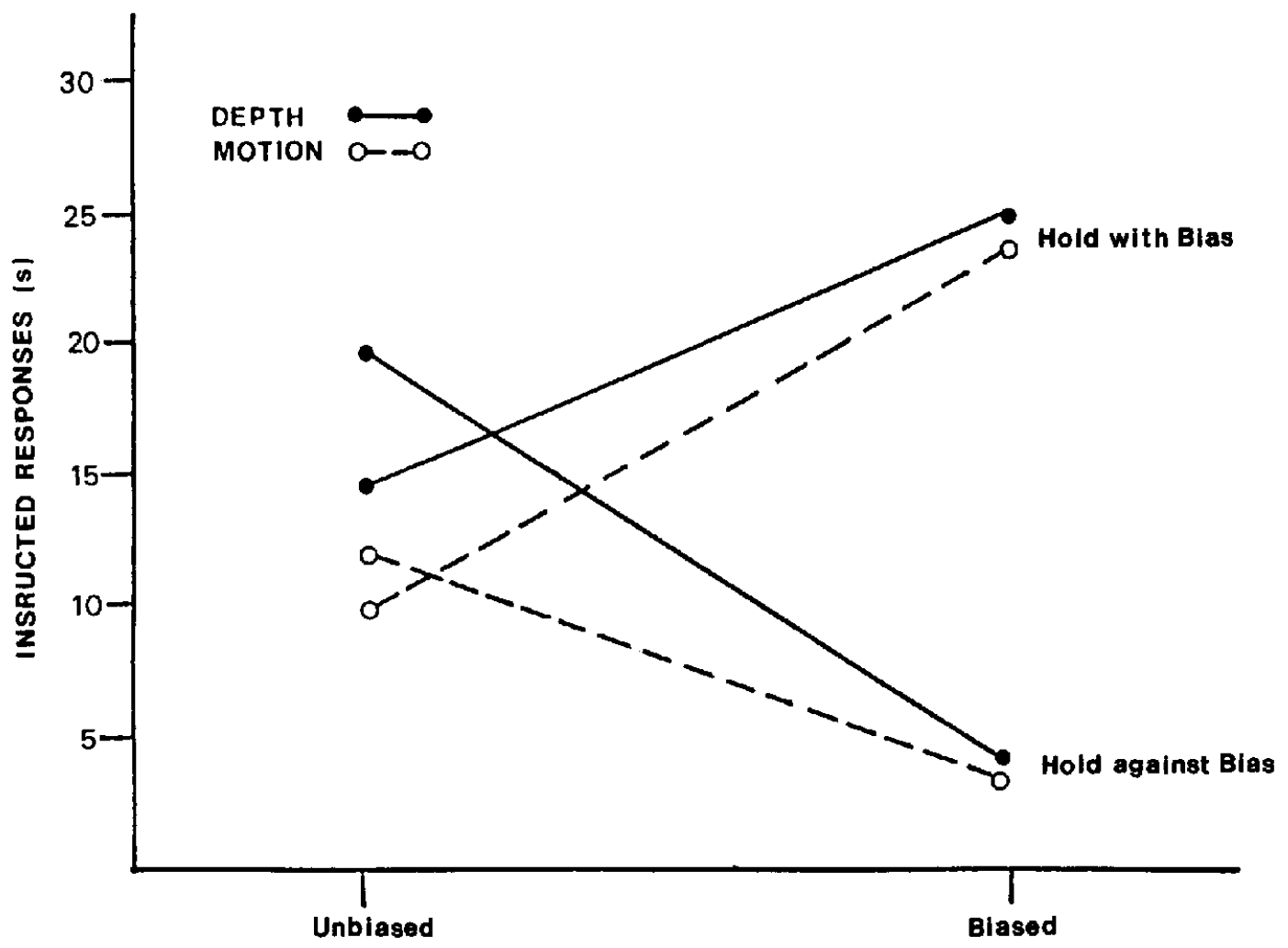

STEREOGRAM BIAS

Figure 2. The mean duration of instructed response during a 30-s trial. (The parameters are instructions to hold the depth favored by the biased stereogram or the alternate depth. Unbroken line represents depth; broken line represents motion.)

\section{Results}

As can be seen in Figure 2, instructed responses indicated that the hold instruction influenced perceived depth to a degree influenced by stimulus bias, $F(1,7)=58.71, p<.0001$ : The difference between the subjects' ability to hold the two depth organizations was greater on the biased (24.1) than on the unbiased stereogram (-1.6). Instructed responses did not differ across response type, $F(1,7)=2.77$. A main effect of hold instruction indicated that the anaglyphs were appropriately biased: Instructed response durations consistent with bias (20.4) were longer than those inconsistent with bias $(9.2), F(1,7)=28.15, p<.002$.

As shown in Figure 3, the intention index showed stronger intention effects on the unbiased (.32) than on the biased (.13) stereogram, $F(1,7)=6.2, p<.05$, supporting the Bias $\times$ Hold interaction in the instructed responses. However, unlike the instructed responses, the intention index was smaller with motion responses (.13) than with depth responses $(.31), F(1,7)=5.61$, $p<.05$.

As can be seen in Figure 4, flat-time measures showed that the differences between the intention index and the instructed responses are not due to subjects' labeling an unfused stereogram consistent with hold instructions, inasmuch as flat time did not differ for motion responses (7.7) and depth responses (5.5), $F(1$,
7) $=1.43$. Flat time did vary, however, across the unbiased and the biased stereogram, $F(1,7)=7.2, p<.04$.

\section{Discussion}

Both instructed responses and the intention index suggest that the organization fitted to the reversible display can be determined jointly by stimulus constraints and hold instruction, an effect obtained previously with two-dimensional and three-dimensional cubes (Hochberg \& Peterson, 1985; Peterson \& Hochberg, 1983): Intention effects on the unbiased stereogram are larger than those on the biased stereogram. Flat time also varied with stimulus type, an effect that may represent stimulus differences as well as intentional effects.

Furthermore, neither flat time nor instructed responses varied with response type, although the intention index did, suggesting that more uninstructed depth was measured through motion responses than through depth responses. This difference may either indicate viewers' reluctance to report uninstructed depth, or it may be due to procedural differences between depth and motion trials: On depth-response trials, subjects were stationary, whereas on motion-response trials they were moving. The subjects' motion may have affected perception by causing a change 


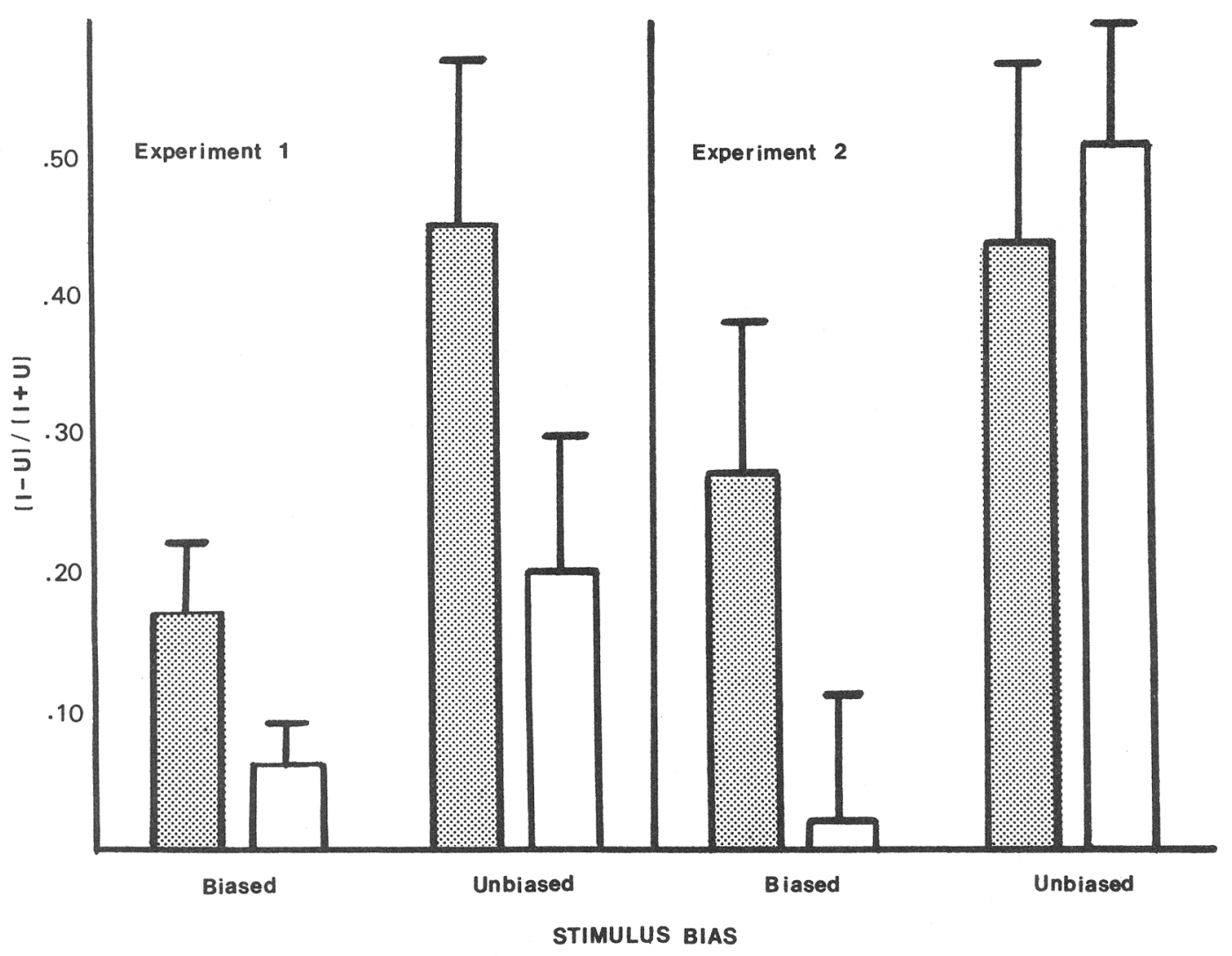

Figure 3. Intention index, $(I-U) /(I+U)$, as a function of stimulus bias, for Experiments 1 and 2. (Shaded and unshaded bars are depth and motion responses, respectively.)

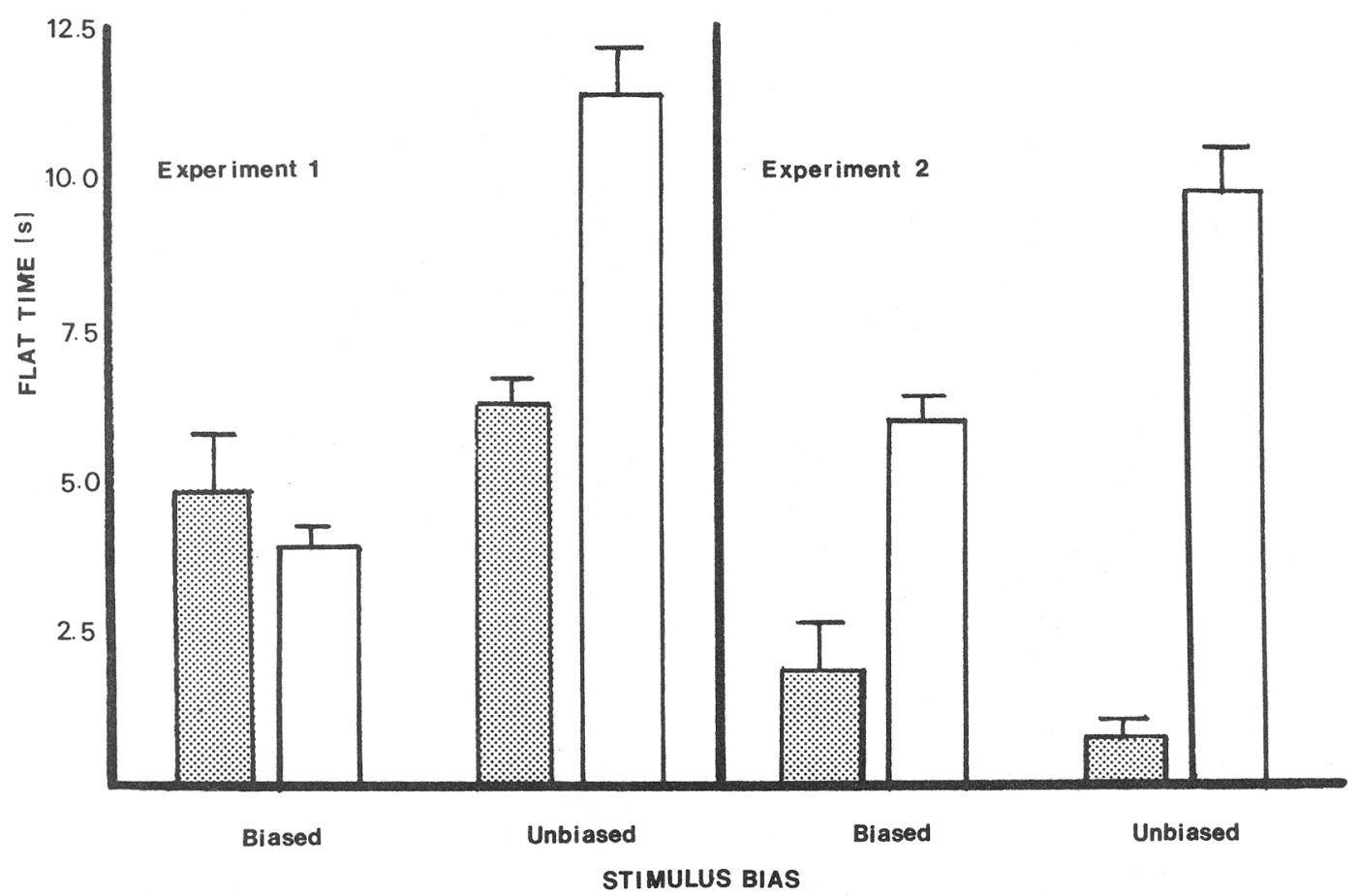

Figure 4. Flat time as a function of stimulus bias in Experiment 1 and 2. (Shaded and unshaded bars are depth and motion responses, respectively.) 
in the effective stimulus, by interfering with the intention task, or by influencing the kind of eye movements occurring during a trial. To eliminate the first two of these possibilities, in Experiment 2 moving subjects responded about bath depth and motion.

In addition, the possibility that intention effects are mediated by vergence changes was explored further in Experiment 2 . Recent evidence indicates that fusion time varies with the distance in stereospace between the plane of fixation and the apparent location of the region depicted in depth (Chung \& Berbaum, 1984). In the opposed-set procedure, therefore, subjects may comply with the instructions to hold front or back by converging or diverging their eyes, respectively, rather than through the selection of different cognitive solutions to fit to the display. If peripheral adjustments such as these are the only means by which instructed intention affects object perception, perceptual organization may indeed be motivationally impenetrable.

In Experiment 1, subjects were asked to keep the nonius cross aligned, but their performance was not monitored. In Experiments 2 and 3, the nonius cross was replaced by a vernier nonius, and subjects' vergence position was monitored through responses about vernier offset. In addition, vergence changes occurring with lateral movement due to the different axes of rotation of the head and eyes (Post \& Leibowitz, 1982) were eliminated by requiring subjects to move vertically.

Extent of subject movement was controlled also. Even though Gogel (1982) has shown that vergence changes occurring as a function of viewers' lateral position are too small to account for illusory concomitant motion, such slight changes in vergence may contribute to viewers' ability to hold one depth organization of a reversible display. Suppose, for example, that divergence occurring at one lateral (or vertical) extreme favored the fusion of the back organization. If so, subjects might quickly learn to avoid large excursions when holding front and seek large excursions when holding back. Inasmuch as the extent of the subjects' motion was unrestricted in the first experiment, this remains a plausible hypothesis about how instructed intention affected organization. Accordingly, in Experiment 2, the rate and extent of vertical movement were controlled by the use of a viewing slit moving through a distance preset by the results of the following preliminary experiment.

\section{Preliminary Experiment}

Three subjects (the author and 2 naive subjects) participated in a method of adjustments procedure (Engen, 1971, pp. 2023) to determine that extent of vertical excursion at which viewers could confidently report both (a) no misalignment of parts of the nonius and (b) illusory motion of the central square in the direction appropriate to the hold instructions. On 12 counterbalanced trials, while holding the central square in front or in back, subjects moved vertically with respect to the display. They fixated the nonius throughout and paid primary attention to the alignment of the nonius lines. Viewers began by making large excursions and gradually decreased their extent of movement until they reached an excursion at which the double criterion above was achieved. Their movements were videotaped, and the average excursion during which the double criterion was met was measured. This was taken as the extent at which one could hold the central square in one depth organization and perceive the illusory motion without vergence changes.

As seen in Table 1, all 3 reached a point where they reported no misalignment of the nonius lines concomitant with the illusory motion of the central square. The grand mean of this excursion was used as the extent of the vertical movement for the subjects in Experiment 2, thereby minimizing the potential vergence changes due to subject movement.

In addition to being constrained to move a particular vertical distance on each trial, subjects in Experiment 2 reported on whether the parts of the nonius appeared aligned before and after each trial. If vergence changes are the mechanism through which instructed intention affects perceptual organization, convergence changes would be expected to accompany hold front instructions and divergence changes should accompany hold back instructions.

\section{Experiment 2}

\section{Method}

Subjects. Eight graduate students at the State University of New York at Stony Brook were paid to participate in this experiment. Four were psychology graduate students. All had normal or corrected-to-normal vision.

Materials. Black and white stereograms corresponding to the anaglyphs used in Experiment 1 were superimposed through rear projection through orthogonally polarizing filters onto a Freen screen that maintained polarization. The room was dark, illuminated only by the light from two projectors behind a large wooden frame surrounding the projection screen. The entire stereogram subtended $6.5^{\circ}$, the central region subtended $2.7^{\circ}$, and the disparity between the central square and the surround was $8^{\prime}$ for each alternative depth.

Nonius lines were drawn on identical white circular fields. One was positioned on the left eye's view, the other on the right. These fields were superimposed on the surround of the stereogram just above the central square, again serving as a stimulus for convergence on the plane of projection. The nonius lines were each straight line segments about $2^{\prime}$ of arc wide and $17^{\prime}$ of arc high. When projected, the top and bottom nonius lines were aligned.

A viewing slit $18 \mathrm{~cm}$ wide and $6 \mathrm{~cm}$ high was cut from a piece of plywood, $61 \mathrm{~cm}$ wide and $35 \mathrm{~cm}$ high. A collar 8 in. deep around the opening of the viewing slit excluded objects other than the projection screen from the subjects' view. This apparatus was hung like a pendulum from a frame so that it was positioned directly in front of the subjects at a distance of $180 \mathrm{~cm}$ from the projection screen. In order to keep the viewer's extent and rate of vertical movement constant across conditions, the viewing slit was moved up and down by a system of pulleys to a metronome beating at $0.77 \mathrm{~Hz}$. The duration of each trial was clocked on a Hunter timer (Model 1520). The subjects' responses were accumulated on two Durgin and Browne, Inc. timers (Model LT BX 6A).

Procedure. Subjects viewed the stereograms while wearing orthogonally polarized goggles. During a first session each subject's depth preference was determined as in Experiment 1; for 6 of the 8 subjects, the biased stereogram was biased toward front. Subjects then practiced reversing the stereograms for $20 \mathrm{~min}$. Half practiced with the unbiased stereogram, half with the biased stereogram. They were given no fixation instructions for the first $10 \mathrm{~min}$ of practice. From there on, they practiced reversal while fixating the nonius. During a second session, occurring on another day, subjects practiced reversing the stereogram for 10 min. Next, subjects were told that they would be participating in twenty-six 20-s trials during which they would move vertically behind the viewing slit to the beat of a metronome while viewing a series of stimuli. 
Table 1

Mean Extent of Excursion (in $\mathrm{cm}$ ) at Which Double Criterion Was Reached

\begin{tabular}{lllllll}
\hline & \multicolumn{2}{c}{ Hold front } & & \multicolumn{2}{c}{ Hold back } \\
\cline { 2 - 3 } \cline { 6 - 7 } Subject & $M$ & & & & & \\
\hline & 7.31 & 1.54 & & 7.38 & 1.31 \\
GS & 7.21 & 0.43 & & 7.70 & 0.39 \\
SR & 6.14 & 0.90 & & 8.73 & 0.48 \\
MP & & & & & \\
\hline
\end{tabular}

Note. Grand $M=7.4$.

Hold and fixation instructions were the same as in Experiment 1 with one addition: Immediately after receiving the opposed-set instructions for a particular trial, subjects reported on the alignment of the top and bottom line of the nonius and on the depth organization seen at that moment. Alignment reports were magnitude estimates of the amount of displacement of the bottom line relative to the top line, with line width as the standard. Next, the clock was started and the experimenter began moving the viewing slit. The end of the trial was signaled when the experimenter said "nonius" and stopped moving the viewing slit. At this signal, the subject again reported on both alignment of the nonius and apparent depth of the central region.

Subjects knelt on a padded stool and pressed one of two bottons on hand grips anchored to a table in front of them. They responded about depth by pressing one button whenever the central square appeared to be in front of the surround and the other whenever it appeared behind the surround. If the display appeared flat or if they could not determine the depth organization, they pressed neither key. Responses of motion in the same or opposite direction were obtained in a similar manner.

Subjects were given two practice trials to accustom themselves to moving vertically in time with the viewing slit and pressing buttons to indicate what they saw. They then participated in three blocks, each consisting of eight trials, two with each hold instruction combined with each stimulus. During each block, viewers responded about either apparent depth or illusory motion. Blocks were run in alternating order; half the subjects responded about motion first, the other half about depth. The first block served as further practice and was not included in the analysis. Stimulus presentation was counterbalanced within and across subjects.

\section{Results}

Data were analyzed as in Experiment 1. As shown in Figure 5 , instructed responses again showed an interaction between hold instruction and stimulus bias, $F(1,7)=59.08, p<.0001$. In addition, instructed responses were greater with instructions to hold with bias $(11.7)$ rather than against bias $(7.8), F(1,7)=$ $19.56, p<.004$. Unlike Experiment 1, instructed responses were larger with the depth response (12.2) than with the motion response (7.3), $F(1,7)=10.5, p<.02$. A three-way interaction, Response Type $\times$ Bias $\times$ Hold, showed that the difference between depth and motion responses occurred mostly on the unbiased stereogram: Viewers asked to hold against bias (direction defined by the biased stimulus) reported more success with depth responses (18.2) than with motion responses $(8.3), F(1,7)=9.14$, $p<.02$ (see Figure 5).
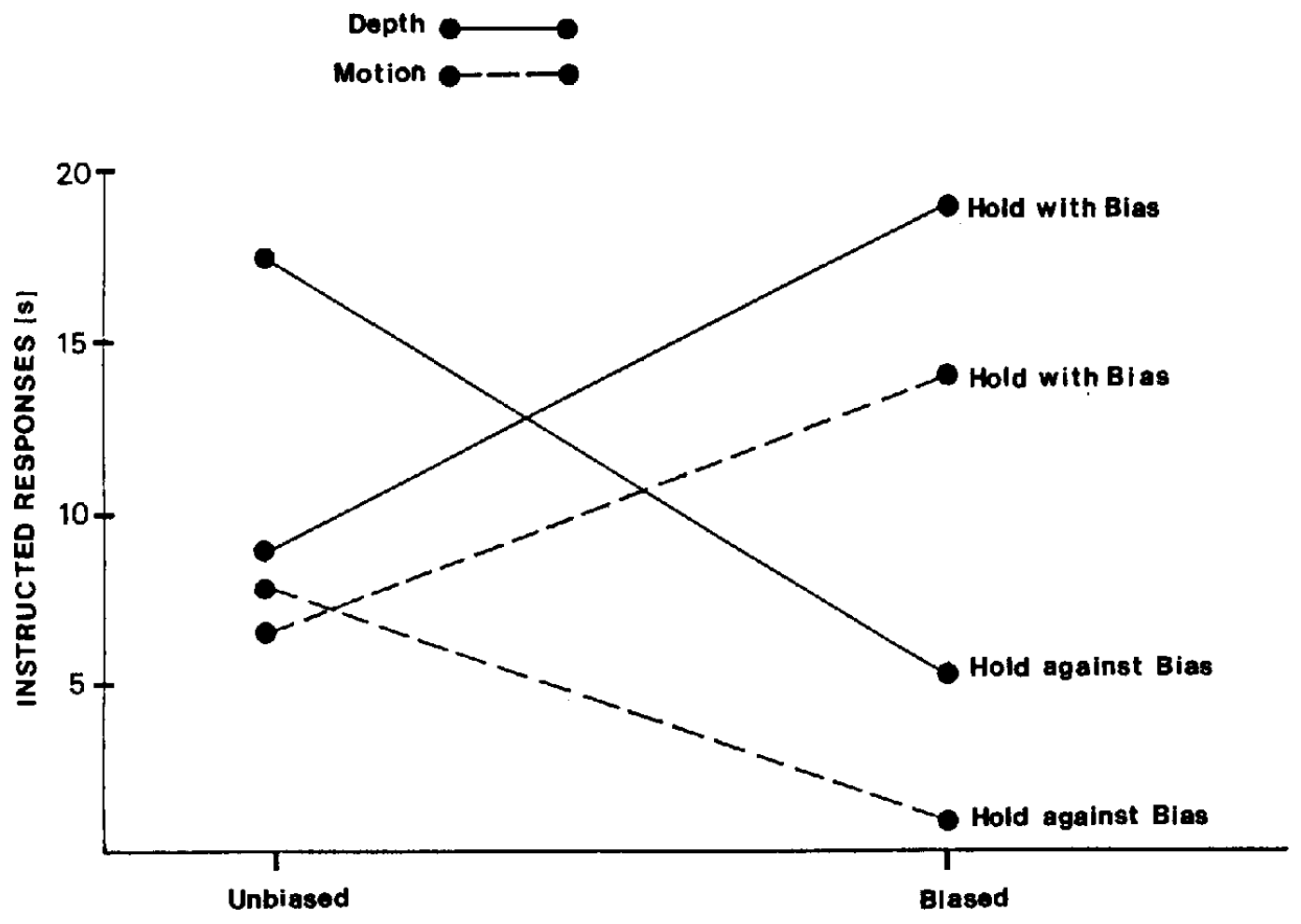

STEREOGRAM BIAS

Figure 5. The mean duration of instructed response during 20-s trials. (Parameters represent instructions to hold the depth favored by the biased stereogram or the alternate depth.) 
As shown in Figure 3, the intention index again supported the Bias $\times$ Hold interaction shown by the instructed responses: Intention effects on the unbiased stimulus (.45) were greater than on the biased stimulus $(.15), F(1,7)=14.91, p<.007$. However, the intention index showed no main effect of response type, $F(1,7)=2.39$, although a Bias $\times$ Response Type interaction showed that depth and motion responses differed on the biased stereogram but not on the unbiased stereogram, $F(1,7)=7.07$, $p<.04$.

Once again, flat-time measures shed light on the different effects obtained with the instructed responses and the intention index. As seen in Figure 4, more flat time occurred with motion responses (derived from failure to report motion) (7.9) than with depth responses $(1.3), F(1,7)=13.03, p<.009$ (see Figure 4). However, in a Bias $\times$ Response Type interaction, motion responses showed more flat time on the unbiased than the biased stereogram, whereas the depth response did not, $F(1,7)=7.12$, $p<.04$. Both the main effect of response type and the Response Type $\times$ Bias $\times$ Hold interaction in the instructed responses may be due to these flat-time effects, inasmuch as the intention index for the unbiased stereogram did not differ with response type. Additional analyses revealed that on the unbiased stereogram, subjects reported more flat time on hold back trials (12.4) than on hold front trials $(10.7), t(7)=3.7, p<.01$.

In addition, depth responses showed longer durations of instructed depth when the depth report accompanying the initial nonius alignment report was consistent (18.7) rather than inconsistent (6.6) with instruction, $t(5)=4.5, p<.01$, although motion responses failed to show this effect, $t(5)=.20$.

Eye movement results. The magnitude estimation reports about the misalignment of the nonius lines ranged from no change in alignment to a change equal to the width of the nonius lines ( 2 ' of arc). Reports of no change occurred on $54 \%$ of the depth-response trials and $58 \%$ of the motion-response trials. The mean misalignment across all trials was equal to $24 \%$ of the width of the nonius lines, which corresponds to $0.5^{\prime}$ of arc, and differs significantly from zero, $t(7)=5.63, p<.0005$.

Reports about nonius misalignment were transformed into measures of vergence in the following way: When the bottom line of the nonius was projected to the right eye, reports that it appeared to the right of the top line were taken to indicate that convergence occurred, and reports that it appeared to the left indicated divergence.

For each subject, both the mean instructed responses and a mean intention index were calculated separately for those trials on which nonius reports showed (a) no change in vergence position, (b) a change consistent with an eye movement account, and (c) a change opposite to that predicted by an eye movement account. Repeated-measures $t$ tests showed no differences among any of these vergence positions. Within-subject $n s$ were small and variability was high. ${ }^{1}$

\section{Discussion}

The results of Experiment 2 suggest that intention effects do not occur postperceptually: The difference between depth and motion responses found with the instructed responses appears to be due to the differential amounts of flat time measured through the two types of response. One explanation for this may be that the vertical extent of movement was not sufficient for all subjects to perceive the illusory motion. The extent of movement was determined from a preliminary study with 3 subjects, and although the standard error around the reported mean movement for each of those subjects was small, the excursion at which the double criterion was met differed for the author (MP) and the other subjects (see Table 1). Subjects in Experiment 2 might have seen more illusory motion if the extent of vertical motion had been tailored for each subject.

Depth and motion response differences are not found with the intention index, measuring differences in the relative distribution of instructed and uninstructed responses, except on the biased stereogram. Here, motion responses suggest that instructed intention was unable to overcome the stimulus constraints present in the biased stereogram, whereas depth responses suggest otherwise. The absence of intention effects on the biased stereogram is not surprising, inasmuch as not all stimulus information is expected to be susceptible to changes in viewers' motivations, although a specification of those conditions in which it is would be useful. However, the difference between the depth and motion responses on the biased stereogram may indicate that monocular depth is more easily ascribed to the biased than the unbiased stereogram, or it may be due to the demand character of the opposed-set procedure. However, the intention effects on the unbiased stimulus are no different when measured directly and indirectly, suggesting that intention can influence processes integral to perceptual organization, and not merely response bias.

As in Experiment 1, the unbiased stereogram appeared to be flat more often than the biased stereogram, but here it was more likely to look flat when subjects tried to hold back than front. suggesting that flat time is not simply associated with the stimulus, but is a function of intention as well. Further exploration of this unexpected finding may provide information about how instructed intention affects perception.

In addition, even though the subjects in Experiment 2 reported nonius misalignment, the reports did not indicate any consistent change in vergence with hold instruction. That the illusory motion varies as a function of instructed depth without large vergence changes supports Gogel's (1982) claim that oculomotor cues are not the basis for illusory motion. The results reported here need not imply that the perception of depth precedes the perception of motion, however. Depth and motion may be independent, concurrent outputs of a nondivisible stage of processing.

Inasmuch as some of the between-subject tests of eye movement differences were significant, closer examination of vergence changes is warranted-in particular, of those changes occurring during a trial. Accordingly, in Experiment 3 the experimental

\footnotetext{
' However, between-subjects $t$ tests showed some differences: Intention effects obtained with motion responses on the unbiased stereogram were smaller when reports indicated vergence movements opposite to eye movement predictions $(-.28)$ than when they reported either no vergence change (.81), or a change consistent with predictions $(1.0), \mathrm{t}(8)=2.98$, $p<.01$, and $\mathrm{t}(7)=3.17, p<.01$, respectively. In addition, overall betweensubjects intention effects were greater than zero both when reports indicated no vergence change across a trial $(.27), t(7)=2.32, p<.05$; and vergence consistent with hold instruction $(.42), t(7)=2.81, p<.05$; but not when they indicated vergence in the opposite direction $(.20), t(7)=$ -0.97 .
} 
situation was amended so that observers viewed the stereogram with hold instructions, as before, and also with reverse instructions. In addition, the experimental situation was reduced so that, regardless of instruction, trial length was determined by the time until a reversal occurred. In the amended procedure, attention to the nonius lines was stressed above the other tasks.

If the intention instructions are effective in this procedure, the trial durations ought to be longer for hold instructions than for reverse instructions. Furthermore, if vergence movements are essential to the effectiveness of the intention instructions, more convergence movements should occur on hold-front trials than on hold-back or reverse-from-front trials. Likewise, more divergence movements should occur on hold-back trials than on holdfront or reverse-from-back trials.

\section{Experiment 3}

\section{Method}

The subjects from Experiment 2 were paid to participate in Experiment 3 as well. The stimuli and projection apparatus were also those used in Experiment 2, although only the appropriately unbiased stereograms were used. Subjects viewed the stereograms while sitting at a table and using a chin rest. They responded about depth by pressing a foot pedal connected to a Rogers A6 Timer-Driver card in an Apple Ile microcomputer, and about nonius misalignment by pressing one of two microswitches (also connected to the Rogers card) mounted on the table, one to their right and the other to their left. The key to the right indicated that the bottom line was displaced to the right; the key to the left indicated displacement to the left.

Subjects participated in 64 trials. Four intention instructions were used: Two hold instructions in which subjects were asked to try to hold the central square in front of or behind the surround, and two reverse instructions in which subjects were asked to try to reverse the stereogram from back to front or from front to back. For both hold and reverse trials, the time until reversal was recorded.

Viewers began each trial seated in front of the reversible stereogram with their eyes closed. On signal, they opened their eyes, fixated the nonius, and waited for one or the other depth organization to emerge. When the first depth organization was not the one instructed (i.e., if the central square appeared back when the subjects had been asked either to hold front or to reverse from front), subjects reversed the organization of the stereogram into the proper organization and then pressed the foot pedal to indicate that they had begun following the intention instructions for that trial. On trials where the first organization was that instructed, the subjects pressed the foot pedal immediately. In both cases, the latency to push the foot pedal was recorded.

Subjects released the foot pedal when the stereogram reversed. The duration of foot-pedal depression, time until reversal, was the measure of success at following the hold or the reverse instructions. The total duration of each trial was equal to the sum of the latency and the time until reversal. If necessary, hold trials were terminated by the experimenter after $30 \mathrm{~s}$.

Trials were run in blocks of eight. For each block, subjects viewed the stereogram with only one instructed set. Order of instruction across blocks was counterbalanced within and between subjects.

While the foot pedal was depressed, the subjects' main task was to indicate the alignment of the nonius lines. They were told that following the intention instructions was secondary to responding about the nonius lines: They were not to move their eyes or their attention away from the nonius lines at any point even if this meant that a new organization for the stereogram emerged slightly before they released the foot pedal. If they noticed a change in the alignment of the nonius lines just at the moment of reversal, they were either to delay releasing the foot pedal until they had reported the nonius event or to report the change in alignment at the end of the trial. (These verbal reports of vergence position were scored as having a duration of $0.05 \mathrm{~s}$ for purposes of analysis. This duration was shorter than the shortest duration [0.1 s] of misalignment reported through key presses.)

\section{Results}

Intention instructions effectively controlled responses about depth organization in the amended procedure. Time until reversal was longer for hold (11.5) instructions than for reverse instructions (8.4), $F(1,7)=11.20, p<.02$. In addition, mean time until reversal on reverse trials (8.4) was longer than the mean latency to push the foot pedal on trials where the initial organization perceived was inappropriate for the intention instruction (5.7), $t(6)=3.08, p<.05$.

Responses of nonius misalignment were converted into estimates of convergence $(C)$ or divergence $(D)$. Two vergence indices, $(C-D) /(C+D)$, were calculated for each subject for each instruction for those trials where misalignment occurred ( $n \mathrm{~s}$ ranged from 11 to 16 , median $=15$ ). One index represented the relative duration of convergence and divergence; the other, the relative number of convergence and divergence movements. Positive values of these indices indicate more convergence; negative values indicate more divergence.

Neither vergence index varied with the organization perceived during the trial, $F(1,7)=2.54, p<.16$, for the duration index; and $F(1,7)=1.21$, for the number index. The duration index is close to showing that convergence accompanies front organization and divergence accompanies back (indices equal to .27 and -.13 , respectively), but the within-subject variability is high. Likewise, the interaction between perceived organization and instruction to hold or to reverse is not significant for vergence duration, $F(1,7)=3.37, p<.11$, or number, $F(1,7)=1.3$. Again, there is a hint that the duration index may decrease when subjects try to reverse from front (.23) rather than hold front (.31) and increase when they try to reverse from back $(-.11)$ rather than hold back (-.16). However, as in Experiment 2, the withinsubject variability is high. ${ }^{2}$

Additional analyses on within-subject data showed that the last vergence change before reversal was more likely to be a convergence when the reversal was to front, and a divergence when reversal was to back: That is, a convergence occurred last more often than a divergence on reverse from back trials as opposed to either reverse from front or hold front trials, $t(7)=2.56, p<$ .025 , and $t(7)=2.37, p<.05$, respectively. This was true also for hold back trials as opposed to hold front and reverse from front trials, $t(7)=2.03, p<.05$, and $t(7)=2.72, p<.025$, respectively. These results suggest that the last vergence change

\footnotetext{
${ }^{2}$ Again, between-subjects $t$ tests show some of these effects to be significant: The duration index was smaller on reverse from front trials than on hold front trials, $t(7)=1.95, p<.05$. However, the difference between reverse from back and hold back was not significant, $t(7)=1.0$. As a group, subjects' mean difference in vergence state across the hold and reverse conditions differs for trials on which the front and back organizations are perceived during the trial, $t(14)=1.93, p<.05$. The vergence number index was also smaller on reverse from front than on hold front trials, $t(7)=1.94, p<.05$.
} 
occurs as a reaction to the stimulus rather than as the mechanism of reversal.

\section{Discussion}

In Experiment 3, differential intention instructions resulted in different responses about perceptual organization, but they were not reliably accompanied by different vergence movements. This is consistent with the results obtained in Experiment 2 and with other research showing that fixation changes do not reliably affect the depth perceived in two-dimensional reversible figures (Ellis \& Stark, 1979; Guilford \& Helson, 1929; Washburn, Regan, \& Thurston, 1934).

However, the fact that subjects reversed the depth more quickly when they were not responding about nonius alignment suggests that the nonius task interferes with the intention instructions. The attenuation of the intention effects may be due to the division of attention between nonius and central square or, alternatively, the nonius task may have limited the subjects' control over vergence changes by forcing vergence at the projection plane. Nevertheless, subjects under instructions succeeded in holding depth for durations longer than they took to reverse from one depth into the other, thereby suggesting a residual effect of intention.

Of course, the possibility remains that intention effects occur as vergence changes too small or fast to be detected as vernier offset (although hysteresis phenomena demonstrate that stereo fusion is robust to vergence change, at least when stereograms are unambiguous, Fender \& Julesz, 1967). Another possibility is that intention operates as the differential distribution of spatial attention, such as that involved in detection and search tasks. In the stereograms, disparity discontinuities specifying the two organizations occur in different regions of the display, at the right edge of the projected central region for the back organization, and at the left edge for front. If the resolution of stereoscopic depth is facilitated by disparity discontinuities, as Gillam, Flagg, and Finlay (1984) suggest, attention to different regions of the visual field may influence the resolution of depth (but see Marr \& Poggio, 1979). However, basic procedural differences, such as the use of blocked versus unblocked and long versus short trials, distinguish the methodologies used to examine these two types of attention and limit further speculation about their similarities.

\section{General Discussion}

In the three experiments reported here, instructed intention was shown to affect perceptual organization, a process often proposed to be automatic (Shiffrin \& Schneider, 1977) or stimulus based (Gibson, 1979). The experiments reported here show these explanations to be insufficient: Given constant stimulus information, both depth and direction of illusory motion varied with viewers' instructed intentions. The fact that the perceptually coupled motion varied with intention suggests that these effects are perceptual, rather than postperceptual, and the absence of systematic eye movements suggests that large vergence changes are not the mechanisms of intention.

Two models of the perception of ambiguous depth are compatible with these and previous results (Peterson \& Hochberg, 1983; Hochberg \& Peterson, 1985). In the first model, intention affects the interpretation fitted to the output of early units: When depth information is ambiguous or when motion signals are equivocal (as in three-dimensional cubes) or absent (as in stereograms), higher level processes may determine depth and motion (cf. Braddick, 1980).

In the second model, outputs of early, impenetrable analyzers are weighed together with comparator (von Holst, 1954) outputs and/or with some index of attentional distribution before depth or motion is assigned. This model is similar to other assessments of top-down processing (Palmer, 1975), although no executive system is implied. Here, top-down processing would occur as the addition of evidence favoring one solution or the other, such as might be provided by changes in eye position or distribution of attention, and perceived organization is determined by the weighted sum of the evidence.

Before conclusions can be drawn about the cognitive or motivational penetrability of object organization, however, the mechanisms through which intention affects perception must be identified. If the mechanisms involve adjustments of the eyes or the differential distribution of a form of attention that is purely visual, intention effects may be domain specific, and object perception may still be an encapsulated system (Fodor, 1983), impenetrable to all but visual influences. The experiments reported here suggest a means to begin that exploration.

\section{References}

Braddick, O. J. (1980). Low-level and high-level processes in apparent motion. Philosophical Transactions of the Royal Society (London), Series $B, 290,137-151$.

Chung, C. S., \& Berbaum, K. (1984). Form and depth in global stereopsis. Journal of Experimental Psychology: Human Performance and Perception, 10, 258-275.

Ellis, S. R., \& Stark, L. (1978). Eye movements during the viewing of Necker cubes. Perception, 7, 575-581.

Engen, T. (1971). Psychophysics I. Discrimination and detection. In J. W. Kling \& L. A. Riggs (Eds.), Woodworth \& Schlosberg's experimental psychology (3rd ed., pp. 11-46). NY: Holt, Rinehart and Winston.

Fender, D. H., \& Julesz, B. (1967). Extension of Panum's fusional area in binocularly stabilized vision, Journal of the Optical Society of America, 57, 819-830.

Fodor, J. A. (1983). The modularity of mind. Cambridge, MA: MIT Press.

Fodor, J. A., \& Pylyshyn, Z. (1981). How direct is visual perception? Some reflections on Gibson's "ecological approach." Cognition, 9, 139196.

Foley, J. M. (1980). Binocular distance perception, Psychological Review, $87,411-434$.

Gibson, J. J. (1979). An ecological approach to visual perception. New York: Houghton Mifflin.

Gillam, B., Flagg, T., \& Finlay, D. (1984). Evidence for disparity change as the primary stimulus for stereoscopic processing. Perception \& PSychophysics, 36, 559-564.

Gogel, W. C. (1976). An indirect measure of perceived distance from ocular-motor cues. Perception \& Psychophysics, 20, 419-429.

Gogel, W. C. (1981). The role of suggested size in distance responses. Perception \& Psychophysics, 30, 149-155.

Gogel, W. C. (1982). Analysis of the perception of motion concomitant with a lateral motion of the head. Perception \& Psychophysics, 32 , 241-250.

Guildford, J. P., \& Helson, H. (1929). Eye-movements and the phi phenomenon. American Journal of Psychology, 41, 595-606.

Haber, R. N. (1966). Nature of the effect of set on perception. Psychological Review, 73, 335-351. 
Hay, J. C., \& Sawyer, S. (1969). Position constancy and binocular convergence. Perception \& Psychophysics, 5, 310-312.

Helmholtz, H. von. (1962). Treatise on physiological optics: Vol. III. (Trans. from the 3rd German ed., J. P. C. Southall). New York: Dover. (Original work published 1866)

Hochberg, J. (1956). Perception: Toward the recovery of a definition. Psychological Review, 63, 400-405.

Hochberg. J. (1970). Attention, organization, and consciousness. In D. I. Mostofsky (Ed.), Attention: Contemporary theory and research (pp. 99-124). NY: Appleton-Century-Crofts.

Hochberg, J. (1974). Higher order stimuli and interresponse coupling in the perception of the visual world. In R. B. Macleod \& H. L. Pick (Eds.), Perception: Essays in honor of James J. Gibson (pp. 17-39). Ithaca: Cornell University Press.

Hochberg. J. (1981). On cognition in perception: Perceptual coupling and unconscious inference. Cognition. 10, 127-134.

Hochberg, J., \& Peterson, M. A. (1979, September). The effect of intention on perspective reversals. Described in J. Hochberg, Perceptual intentions and mental structures. Distinguished scientist contribution award address presented at the meeting of the American Psychological Association, New York.

Hochberg, J., \& Peterson, M. A. (1985). Perceptual couples as measures of the role of local cues and intention in form perception. Unpublished manuscript.

Holst, E. von (1954). Relations between the central nervous system and the peripheral organs. British Journal of Animal Behavior, 2, 89-94.

Julesz, B. (1971). Foundations of cyclopean perception. Chicago: University of Chicago Press.

Julesz, B., \& Johnson, S. C. (1968). Stereograms portraying ambiguously perceived surfaces. Proceedings of the National Academy of Sciences, $61,437-441$.

Kahneman, D., \& Treisman, A. (1984). Changing views of attention and automaticity. In R. A. Parasuraman \& D. R. Davies (Eds.), Varieties of attention, (pp. 29-61). Orlando, FL: Academic Press.

Kaufman, L., \& Pitblado, C. B. (1965). Further observations on the nature of effective disparities. American Journal of Psychology, 78, 379-391.

Lawrence, D. H., \& LaBerge, D. L. (1956). Relationships between recognition accuracy and order of reporting stimulus dimensions. Journal of Experimental Psychology, 5I, 12-18.

MacCracken, P. J., Gogel, W. C., \& Blum, G. S. (1980). Effects of posthypnotic suggestion on perceived egocentric distance. Perception, 9. $561-568$

Marr, D., \& Poggio, T. (1979). A computational theory of human stereovision. Proceedings of the Royal Society (London), Series B, 204, 301328.
Mill, J. S. (1865). An examination of Sir William Hamilton's philosophy. London: Longman's, Green.

Ogle, K. N. (1950). Researchers in binocular vision. Philadelphia: Saunders.

Palmer, S. E. (1975). Visual perception and world knowledge: Notes on a model of sensory-cognitive interaction. In D. A. Norman \& D. E. Rummelhart (Eds.), Explorations in cognition (pp. 279-307). San Francisco: W. H. Freeman.

Peterson, M. A. (1984). Measures of selective components in perceptual organization. Unpublished doctoral dissertation, Columbia University, New York.

Peterson, M. A., \& Hochberg, J. (1983). Opposed-set measurement procedure: A quantitative analysis of the role of local cues and intention in form perception. Journal of Experimental Psychology: Human Perception and Performance, 9, 183-193.

Posner, M. I., Snyder, C. R., \& Davidson, B. J. (1980). Attention and the detection of signals. Journal of Experimental Psychology: General, 109, 160-174.

Post, R. B., \& Leibowitz, H. W. (1982). The effect of consequence on the vestibulo-ocular reflex and implications for perceived movement, Vision Research, 22, 461-465.

Pylyshyn, Z. (1981). The imagery debate: Analogue media versus tacit knowledge. Psychological Review, 88, 16-45.

Pylyshyn, Z. (1984). Computation and cognition: Toward a foundation for cognitive science. Cambridge, MA: MIT Press.

Remington, R. W. (1980). Attention and saccadic eye movements. Joumal of Experimental Psychology: Human Perception and Performance, 6 , 726-744.

Richards, W. (1970). Stereopsis and stereoblindness. Experimental Brain Research, 10, 380-388.

Richards, W. (1971). Anomalous stereoscopic depth perception. Journal of the Optical Society of America, 61, 410-414.

Rock, 1. (1983). The logic of perception. Cambridge, MA: MIT Press.

Schneider, W., \& Shiffrin, R. M. (1977). Controlled and automatic human information processing: I. Detection, search, and attention. Psychological Review, 84, 1-66.

Shiffrin, R. M., \& Schneider, W. (1977). Controlled and automatic human information processing: II. Perceptual learning, automatic attending, and a general theory. Psychological Review, 84, 127-190.

Washburn, M. F., Regan, C., \& Thurston, E. (1934). The comparative controllability of the fluctuations of simple and complex ambiguous perspective figures. American Journal of Psychology, 46, 636-638.

Received February 7, 1985

Revision received September 12, 1985 